\title{
RETIENAS: Real Time Intranet Based Entrance Examination System at Institute of Engineering
}

\author{
Babu Ram Dawadi ${ }^{1}$, Tri Ratna Bajracharya ${ }^{2}$, Ram Chandra Sapkota ${ }^{3}$, Subarna Shakya ${ }^{4}$ \\ Arbind Kumar Mishra ${ }^{5}$, Bharat Raj Pahari ${ }^{6}$, Daya Sagar Baral ${ }^{7}$, Aman Shakya ${ }^{8}$, \\ Sanjeeb Prasad Panday ${ }^{9}$, Arun Kumar Timalsina ${ }^{10}$, Shashidhar Ram Joshi ${ }^{11}$ \\ Institute of Engineering, Tribhuvan University, Pulchowk, Nepal \\ 1, 4, 7, 8, 9, 10, ${ }^{11}$ Department of Electronics and Computer Engineering \\ ${ }^{2,3}$ Department of Mechanical Engineering \\ ${ }^{6}$ Department of Civil Engineering $\mid{ }^{5}$ Department of Electrical Engineering \\ Corresponding authors: ['baburd, ${ }^{1}$ triratna]@ioe.edu.np]
}

Received: Nov 5, $2017 \quad$ Revised: Dec 10, $2017 \quad$ Accepted: Dec 15, 2017

\begin{abstract}
Accepting the benefits of automation and to avoid the existing issues in semi-automated entrance examination system, Institute of Engineering (IOE) had decided to move into fully automated computer based entrance examination system in 2013. IOE had implemented automatic answer sheet scanning and processing system since 2004 and started online based application since 2009. From the year 2014, IOE has started fully computer based entrance examination system viz. Real Time Intranet based Entrance ExamiNAtion System (RETIENAS) at its resourceful centers (ICTC) at Pulchowk. The fully managed and hassle free application processing system of IOE entrance examination allows applicants to simply apply and receive admit card with specified time slot with the their own choice from online registration system. In this paper, we presented the conceptual framework with functional architecture of RETIENAS. Performance evaluation of RETIENAS shows better result for the allocated shift consisting of 250 examinees during the exam. We have also elaborated on the server and networking infrastructure with software system architected for RETIENAS. IOE need to continuously step ahead towards the excellence by improving the entrance system by addressing the challenges and follow the future directions.
\end{abstract}

Keywords: BE/BArch, entrance exam, IOE, automation, quality, RETIENAS

\section{Introduction}

IOE is the government owned oldest institution in Nepal providing quality education in the engineering domain since 1972. As being the top ranked institute under the national university, IOE is continuously improving its education system and research culture with its major features to have dedication on quality education to achieve the world class standards in engineering education. As a public institution, it provides equal opportunities for all qualified citizens to enter into the university education. These days, there are generally more than 12,000 competitive applicants in the entrance exam of bachelors program and more than 2,000 applicants in its M.Sc. entrance exam. Hence less than $32 \%$ applicants are selected for the admission. The increase of entrance applicants every year and the increasing burden in the manual processing of the entrance exam enforces IOE to step into the automatic exam operation and result processing system. 
This paper is organized as follows: Section 2 and 3 highlight the history of IOE and entrance examination conducted by IOE since its establishment. Section 3 and its subsections present the automation concepts and overview of RETIENAS, while section 4 presents in details about the operational framework of RETIENAS, software architecture, infrastructure implementation approach and security aspects of the system. Section 5 presents the performance analysis results of software and the network operation. Possible challenges are discussed on section 6 including future recommendations following the conclusion in section 7 .

\section{History of IOE}

History of engineering education in Nepal can be traced since 1942 AD, when Technical Training School was established [1]. Engineering section of the school offered only trade courses and civil sub-overseers programs. In 1959, Nepal Engineering Institute, with the assistance of the government of India, started offering civil overseer courses leading to Diploma in Civil Engineering. The Technical Training Institute established in 1965, with the assistance from the Government of Federal Republic of Germany, offered technician courses in General Mechanics, Auto Mechanics, Electrical Engineering and Mechanical Drafting. In 1972, the Nepal Engineering Institute at Pulchowk and the Technical Training Institute at Thapathali were brought together under the umbrella of the Tribhuvan University (TU) to constitute the Institute of Engineering (IOE). Nepal Engineering Institute and the Technical Training Institute were renamed as Pulchowk Campus and Thapathali Campus respectively. Since then, IOE has expanded considerably. The technician programs in Electrical, Electronics, Refrigeration/Air-conditioning Engineering were started in the Pulchowk Campus, with the assistance from UNDP/ILO in 1972. The Architecture Technician program was started by the IOE in its own effort in 1973. As a first Bachelor's Degree level course in Engineering in Nepal, B. E. Civil Engineering was started in 1984 in its own effort. In 1994, with the assistance of the World Bank, the Swiss Government, and the Canadian Government, through Engineering Education Project (EEP); Bachelor Degree level courses in Engineering were extended to Electronics \& Communication and Electrical Engineering. In 1995, Mechanical engineering and Architecture were started in the Pulchowk Campus. Beyond the project period, IOE extended additional bachelors programs in following years. Bachelor's Degree program in Computer Engineering, from 2001, Bachelor's Program in Agriculture Engineering were started at Purwanchal Campus. In 2006, Bachelor's Program in Industrial Engineering in 2015, Bachelor's Program in Automobile Engineering were started at Thapathali Campus. From the year 2014, all the diploma programs were phased out and the IOE concentrated its programs spanning from Bachelor through Master to Ph.D. levels.

In 1996, Pulchowk Campus with support from the Norwegian Government has started M.Sc. Courses in Urban Planning, Structural Engineering, Environmental Engineering and Water Resources Engineering. Pulchowk Campus has also started M.Sc. courses in Renewable Energy Engineering, Geotechnical Engineering, Information and Communication and Power System Engineering effective from December, 2001. Pulchowk Campus, with support from the Norwegian Government, has started M.Sc. Courses in Sustainable Water Sanitation, Health and Development from 2007, Technology and Innovation Management from 2010. This campus has also started master program in Energy Systems Planning and Management, Transportation Engineering and Disaster Risk Management from the academic year 2011/12. Institute has also started regular Ph.D. admission from academic session 2010/11 in all departments. Additional master programs - Climate Change and Development in 2013, Material Science and Engineering in 2014, Energy for Sustainable Social Development in 2015- had been started at Pulchowk Campus. Similarly, new master programs in Electrical Engineering in Distributed Generation, Civil Engineering in 
Infrastructure Engineering and Management, and Master program in Communication \& Knowledge Engineering have started at Paschimanchal Campus Pokhara from the academic year 2014AD. Earthquake Engineering in 2014, Mechanical Design and Manufacturing in 2016 were also started at Thapathali Campus. Mechanical Design and Engineering and Energy Efficient Building in 2016 were started at Pulchowk Campus in 2016.

\section{History of IOE Entrance Examination}

IOE started its entrance exam at its diploma, bachelor and master's program since its establishment. IOE has always focused towards meritorious candidates for the admission at its BE/BArch and M.Sc. programs by taking standard entrance examination every year since its establishment. With the growing popularity and quality focus of the institute, the number of applicants in the entrance examination is increasing every year. The recent six years application status is shown in Fig. 1. It is obvious that increasing population density of Nepal leads to the increase in the number of applicants at IOE entrance examination. Similarly, with the addition of new programs and colleges, the total intake capacity of IOE is also increasing. Fig. 2 presents the total available seats for every campus/colleges of IOE. This indicates that almost 30\% applicants can get opportunity for the admission in the bachelor programs $[4,5]$.

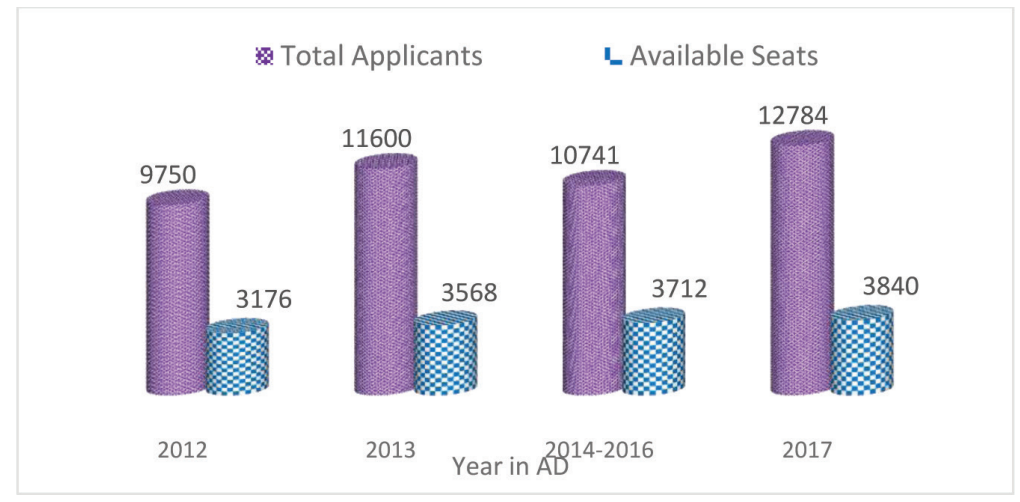

Fig. 1: Year wise applicants and available seats at IOE

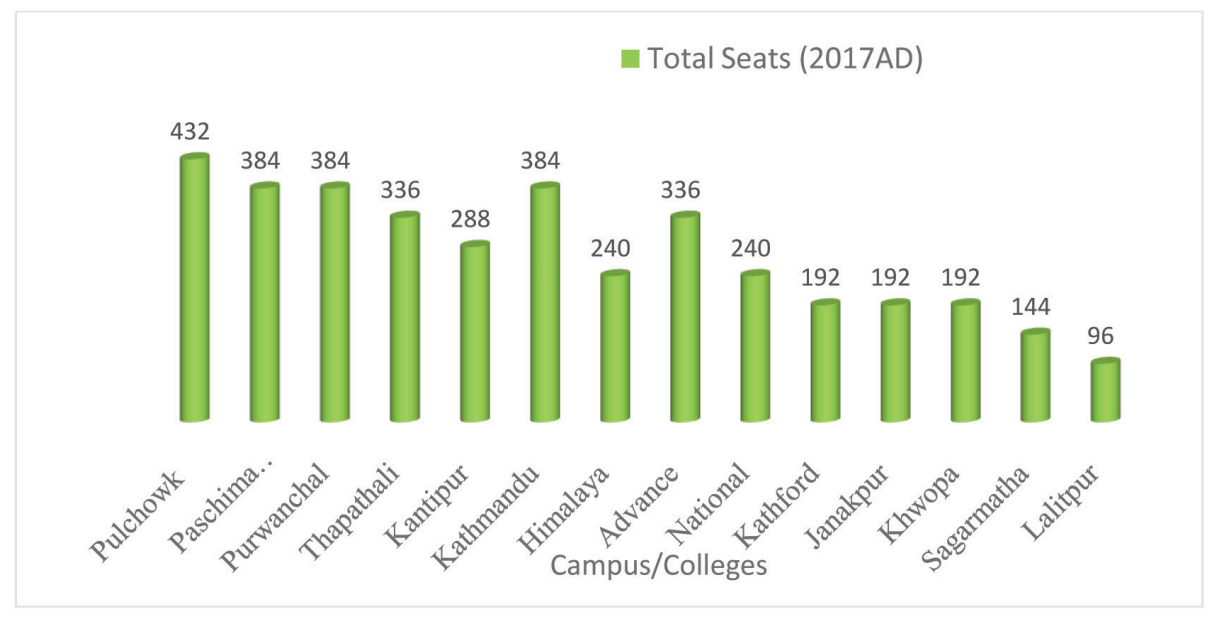

Fig. 2: Available admission seats of IOE campuses/Colleges [2]

The manual processing of application form submission, admit card generation and distribution, 
question sets processing, answer sheet processing with exam center management were a burden and challenges for IOE to have error free result processing. Since 2004, IOE developed automatic answer sheet scanning and processing system but the application form processing was manual. The online based application form submission and processing with automatic answer sheet scanning and result processing was started since 2009 as a semi-automatic examination system. After IOE decided to move its entrance exam into the fully automatic computer based system, then in 2013, IOE conducted two level of entrance exam: first phase in fully computer based and second phase in semi-automatic conventional method same as applied in the previous years. IOE found that the fully automatic computer based entrance exam is more robust, error free and efficient as compared to the manual and semi-automatic methods used in the past. From the year 2014, IOE has started as a research and development project so called Real Time Intranet based Entrance ExamiNAtion System (RETIENAS) to carryout fully computer based entrance examination system at its resourceful centers at Pulchowk. The fully managed and hassle free application system of IOE entrance examination allows applicants to simply apply and receive admit card with specified time slot from online registration. Similarly, after the automation of entrance system, IOE has also conducted another research and development project viz. Centralized Admission System of IOE

(CASI =>काजी) [3] offering fully merit based admissions at its constituent campuses and affiliated colleges.

\section{Entrance Exam Automation: Conceptual Framework}

Fig. 3 shows the non-automated and automated activities under the process of semi-automatic entrance examination management at IOE before applying the fully automated entrance system. It shows that the automated processes like online application form processing and result processing frequently require manual interventions, while all other activities like question setting, printing in the press, delivery of question in the exam centers, taking exam, coding, result publications are manual and more challenging from security perspective too.

Manual processing increases the management complexities of the exam basically in the scenario where the applicants are increasing every year. Security and secrecy are the two major factors that measure the credibility of the examination system. It is hard to ensure security and secrecy in the manual exam processing.

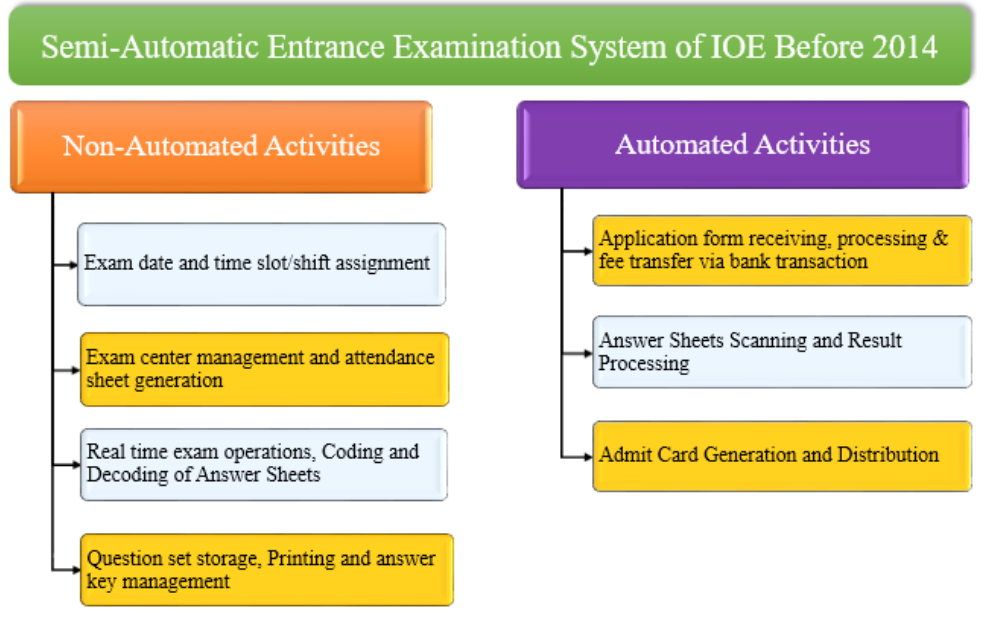

Fig. 3: Module status of semi-automatic entrance exam of IOE 
To avoid the existing challenges and issues of entrance examination, fully automated examination system was conceptualized and implemented by IOE. Fig. 4 presents the features of RETIENAS whiles its operation and technical implementation framework is discussed in section 4 of this paper. The major functionalities of RETIENAS are as follows:

- Online application form registration, processing and admit card generation.

- Incorporated with banking system for application fee payment transfer and automatic voucher processing.

- Applicants themselves choose their exam date and time slot as per their interests.

- Computer based examination - Machine intelligence

- Fully secured intranet system designed with redundancy and threat management

- Hierarchical level of security access privileges form super admin to power admin to system operator

- Automatically and randomly generate password for examinee login

- Automatic import of questions with figures \& formulas and the options.

- Fully managed and secure question bank that can store millions of questions of units and subunits according to given standard syllabus.

- Random generation of questions for every exam slot and locking features of every questions

- Real time clock synchronization between clients and server during exam

- Featured with automatic result processing immediately after completion of exam slot.

- Scalable \& flexible so that expandable to take exam from multiple LANs over geographical distance

In fact this automation minimizes the drawbacks of pervious system, however it still has certain limitations which are briefly highlighted below with its possible steps to be addressed in the future.

- Limited infrastructure: due to limited number of computers in the dedicated labs within the secure intranet system, IOE is still not able to take exam of all applicants at once in a single day. To achieve this, it is required to increase the infrastructure resources towards distributed networked environment so that applicants shall appear in the exam form multiple locations.

- Security threats: however the system is designed with sufficient security, there is always a chance to have attack in the network and applications. For which, IOE has to design and implement the disaster recovery center together with its own CERT (Computer Emergency Response Team) so as to handle the possible disaster in the future.

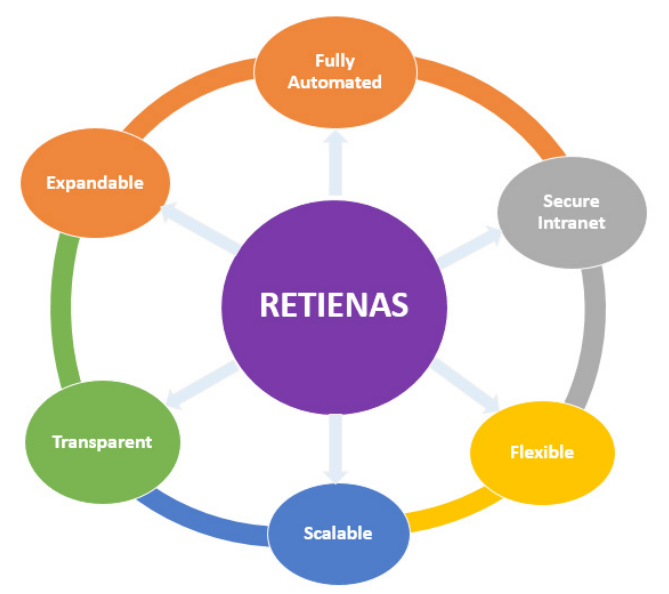

Fig. 4: Features of RETIENAS 
- System Maintenance and Upgrades: with the changing technologies on software and networking paradigm, there is always a challenge for the maintenance and upgrade of the system developed.

- Human Resources: Exam operations like invigilators and other management and control requires highly professional technical human resources. Management of skilled human resource for this real time computer based exam is a challenge for which IOE has to empower existing faculty/staffs with required training/workshop.

\section{Operational Framework of RETIENAS}

The overall functions of RETIENAS is depicted by the process flow diagram in Fig. 5. This system allows entrance applicants to do everything from his/her home internet except the one has to appear in the computer based examination over the dedicated computer center at IOE Pulchowk. Once the entrance exam board of IOE announces the application call with necessary details, applicants deposit the application fee to the entrance board's account from any branch of the bank. The banking system automatically transfers the voucher details (applicant's name, date of birth, deposit date \& deposited amount) via SSH tunnel to the entrance system server. The voucher number is validated during the application submission by the corresponding applicants. Applicants are provided with progress tracking interface from the public site (http://entrance.ioe.edu.np) where he/she can see application acceptance status by supplying necessary credentials. Applicants can download admit card any time after his/her form is accepted. Examinees shall come directly to the computer based exam center at his/her specified date and time slot that was already set by the applicants themselves. Exam system admin (operators) verifies and accepts the application form unless otherwise there is no mistakes in the application. Timely notification shall be sent to applicants to correct the mistakes if any to the individuals via phone/SMS and/or email. Once the application deadline and form acceptance is completed, lab resource planning is started in which software system automatically assign lab and the computer number for an individual examinee. The system has the facilities to export admit card in bulk that helps to cross verify the originality of examinee as well as print attendance sheets and the seat plan for the corresponding exam slot. One hour before the exam starts, two Super Admins in the presence of entrance exam board follow two level of authentications and supply two separate passphrase into the secure application to generate a question set from the question bank for the upcoming exam shift. After proof reading and verification, Entrance Exam Board releases the question set for the specified shift. There is two level of physical access control and four level of authentication access control in the systems server where access passphrases are separately assigned to the super admins. Therefore access to the system by one or more of the passphrase holders in absence of all is not possible. 


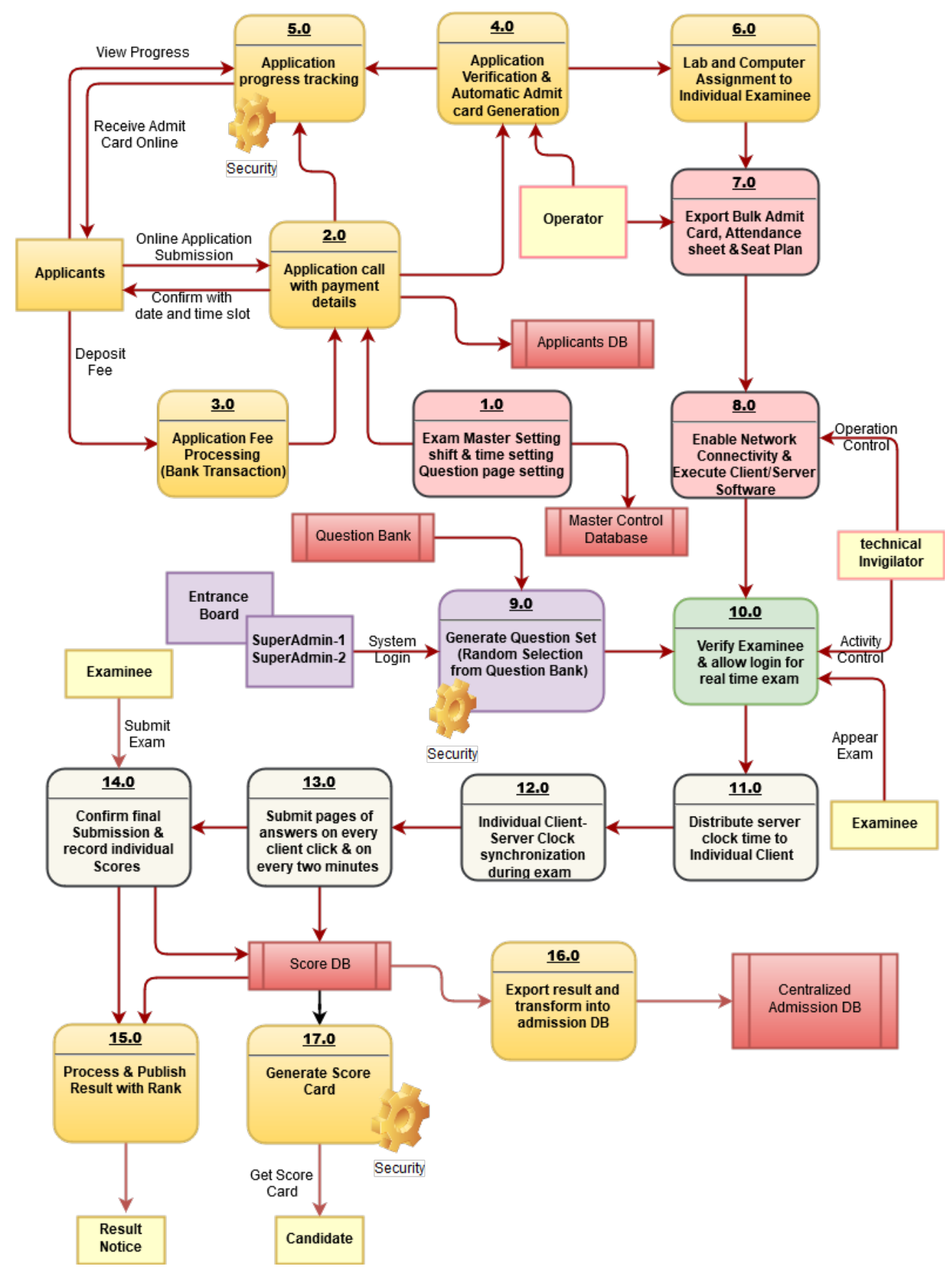

Fig. 5: Process diagram of RETIENAS indicating the major process activities

Technical invigilator and network technicians look after the client-server network connectivity and execute the client software ten minutes before the exam starts, then instruct examinees accordingly. Examinee can't access the computer other than the one assigned to him/her. Keyboard shall be disabled during the exam period. So examinees are allowed only to use mouse and click on the multiple choice answers. When examinee enters into the exam system by supplying his/her own credentials and starts exam, the client clock daemon receives the time stamp value from the server 
clock as Lamport's timestamp [6]. The clock counter in the server is set for two hours and the clock tick continuously decreases. If the client is disconnected, the client synchronized the server clock time once it is recovered. Cristian's clock synchronization algorithm provides the synchronization of real time physical clock, while we implemented the principle that every client computer should be synchronized with server clock time no matter what time it has in the real. The system is implemented on a single LAN over the intranet so that the delays in the time stamp transfer from server to the client is negligible. The delay has to be considered if the system is expanded to be executed form multiple LANs over a geographically far distance. Examinee's photo, clock time and status of answered/unanswered questions will be displayed on the computer screen during the whole exam period. This helps to know the time status to examinee and fully avoid the fake examinee to appear in the exam hall. The record of every click i.e. selection of answers will be written to the server database avoiding the loss of records that does not make difference even when the client machine is crashed. The system has the feature to know the total marks obtained by the examinee immediately after submission of the exam. After completion of whole exam shifts, entrance board processes for the publication of result from the score already obtained during the exam. After applying the negative marking for every wrong answer, the ranking is generated based on the order of total score obtained. For the case of equal marks, then the marks reference on the order of Math, Physics, Chemistry, English and Aptitude of the entrance exam is taken. If the marks is still same, however there is rare chances, then the criteria of ranking is based on the order of average percentage on School Leaving Certificate (SLC), +2 or equivalent of the candidate.

\subsection{Infrastructure Arrangement in the intranet}

IOE has setup dedicated and secure intranet including number of labs at Information and Communication Center (ICTC) Pulchowk to carry out the entrance exam. Fig. 6 shows the networking and server arrangement of the entire system. In which the redundant web and database servers with Unified Threat Management (UTM) system is setup. The Web Application Gateway (ALG) Server running IIS is placed on the demilitarized zone (DMZ), where all the backup and database servers are placed on the private LAN beyond the UTM gateway. Router Access Control List (ACL) and the Server firewall controls the traffic over the public network. The real time exam server located in the private LAN is only accessible form corresponding lab computers. As of 2017, there are nine LABs each containing 30 computers so that entrance board can conduct exam for 270 students in a slot but 20 computers are reserved for backup and hence 250 examinees shall appear in a shift. For 12,000 applicants, the total shifts required to complete the exam is 48 and it takes about 12 days if four shifts are conducted in a day.

\subsection{Software Operations and Management Framework}

The whole system is developed on .NET framework (C\# .NET) on the MVC architecture and the AJAX as a front end applications. Microsoft SQL Server 2012 Enterprise Edition is installed for backend database engine. Number of middleware APIs were developed like for the clock synchronization, automatic import of bank voucher records to the database, recovery of the system after client is crashed, import/export of questions. Firefox internet plugin was imbedded into the client software to login and submit the answer. The developed system was tested, verified and implemented over IPv4 networking infrastructure. The software system is divided into five major packages depicted in the Fig. 7. 


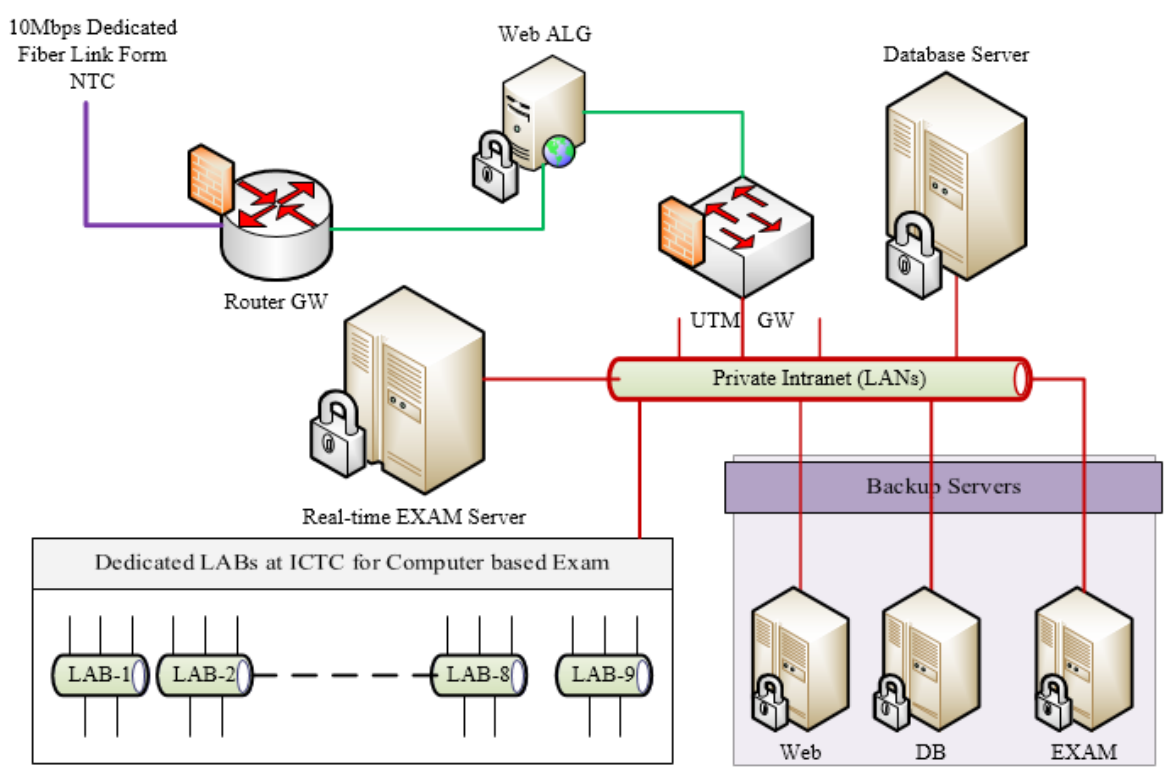

Fig. 6. Server and network connectivity infrastructure for entrance exam

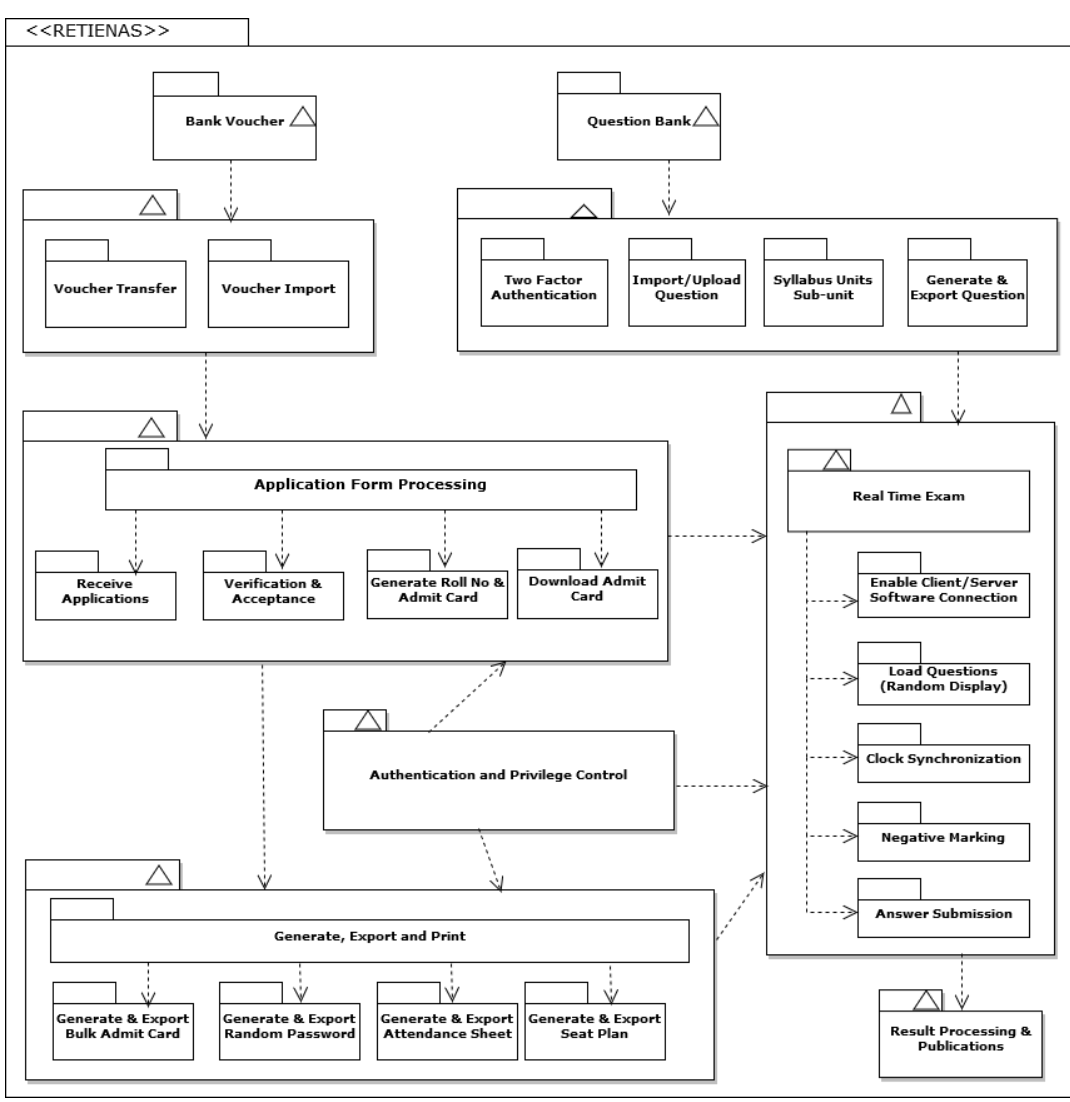

Fig. 7: Package diagram showing the software operation and management of RETIENAS 


\section{System Performance Evaluation}

IOE has successfully developed and implemented this software systems since 2014. Due to the increasing number of concurrent connections on database server, it is required to continuously monitor the server CPU utilization and memory usage ratio of the real time exam and database server. In a shift, at most 250 examinees can appear in the exam. Hence if all examinees click the answer of any question at a time, the concurrent request to exam and database server is just 250 while the MS SQL server 2012 supports at max 32767 user connections. We have recorded the maximum CPU and memory utilization of real time exam server as well as database server during the exam operation consisting of 250 examinees in a shift. The real time exam server equipped with quad core processor and $16 \mathrm{~GB}$ of memory is found to be more than sufficient to handle request processing of 250 examinees. It has only $13 \%$ CUP and $34 \%$ Memory Utilization. While the database server has quad-core processor with $32 \mathrm{~GB}$ of memory and it has CPU and memory usage status of $6 \%$ and $25 \%$ respectively. The performance status shown in the Fig. 8 indicates that IOE can conduct exam with thousands of examinees with this existing infrastructure. The existing infrastructure is easily expandable due to its openness property to be expandable towards distributed networked environment in the future.

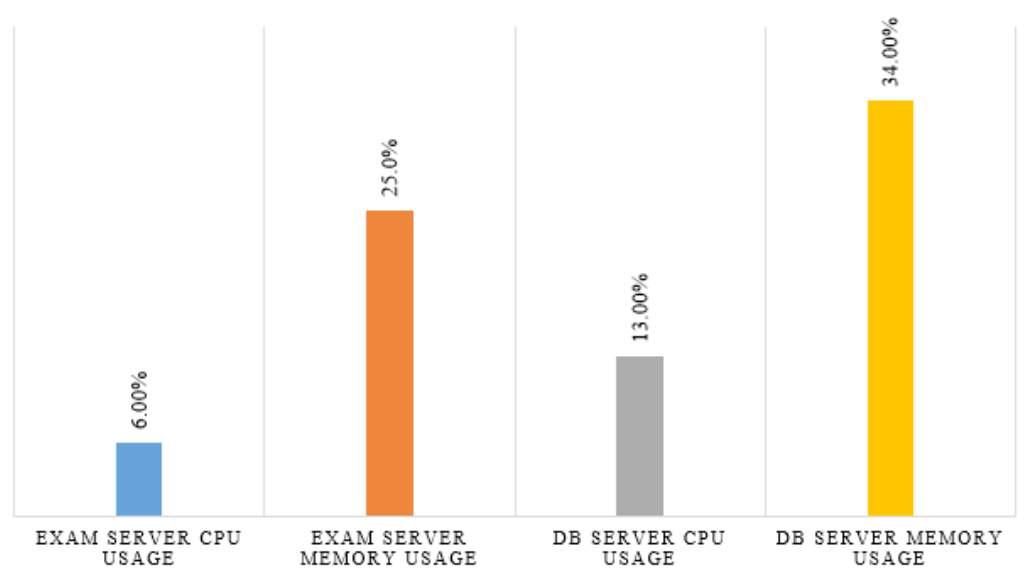

Fig. 8: Maximum CPU and Memory usage Status of Real Time Exam Server \& DB Server

\section{Existing Challenges and Future Works}

Focusing towards the quality of engineering education, IOE is continuously improving the entrance examination system while the timely revision on entrance syllabus is required to be considered to select highly qualified candidates from the large number of applicants. The major challenges on this automated exam system is to execute exam at one shift for all applicants so that the evaluation shall be more exact to judge the students relatively from a single set of question. The major concern is the resource limitation where immediate provisioning of computers and labs for more than twelve thousands applicants and conduct exam on one sitting is almost impossible. However IOE set the policy to add the labs with computers every year. For example the number of computers was increased from 180 to 270 within the year from 2014 to 2017.

Another major concern is to measure the feasibility that whether IOE can conduct the exam from its constituent campuses at Dharan and Pokhara. That will be another milestone for IOE to run the exam at once from multiple locations. For this, lab resource benchmarking of the constituent campuses is required. Because this exam needs to have efficient, reliable and sophisticated 
computers in a networked environment. To achieve this, either it is required to upgrade the existing software and make it distributed or connect those centers using VPN tunnels form Kathmandu. We need to have distributed cache based servers to be synchronized with the centralized servers.

Increasing the examinees in a shift also increases the number of concurrent connections to the servers leading to network congestions. Message queuing system shall be implemented to avoid the possible loss of packets due to congestions. We need to consider towards the design of delay and fault tolerant network. It is also required to consider and optimize the propagation delay in the client server clock synchronization while taking the exam in the distributed environment. In case of client crash, it is required to automatically adjust the time loss of the corresponding examinees once the client connection is recovered. An individual clock daemon shall be maintained in the server process to handle such situation.

For the improved version of the system with better performance, it is recommended to migrate the existing networking infrastructure into SDN and IPv6 operable network so that IOE could achieve more flexibility, scalability, manageability, security, operability and the efficiency with RETIENAS.

\section{Conclusion}

Entrance exam is just a screening test of the eligible applicants and it is considered as a major quality indicator. IOE entrance examination board publishes the result of examinees who secure minimum pass threshold as set by IOE faculty board. Then the eligible candidates themselves have to apply separately to campus/colleges based on their rank secured for admission. We compared the old entrance exam system with currently running entrance system - RETIENAS and evaluated that automation helps towards the excellence in quality of engineering education. The evaluation of server systems, software and network performance shows that the system is flexible and scalable so that IOE shall be able to avoid the existing challenges and achieve the set objectives and recommendations.

Acknowledgement: RETIENAS is a research based project initiated with the objective to select highly qualified candidates through sophisticated computer based entrance examination system for bachelor/master level of engineering study at the Institute of Engineering (IOE), Tribhuvan University. This research was supported by Institute of Engineering, Dean's Office under the project "Reform of Institute of Engineering" with its regular grants on "Steps towards Excellent Academic Environment at IOE".

\section{References}

[1] Bajracharya T (2015), Presentation Document by Prof. Dr. Triratna Bajracharya on Brief Introduction of IOE.

[2] BE/BArch Entrance Exam and Admission Information Directives-2074, http://entrance.ioe.edu.np

[3] Dawadi BR and Baral DS (2017), Towards Automation in the Admission Process as a Tool to Enhance Quality of Engineering Education at Tribhuvan University, Journal of the Institute of Engineering, Nepal, Accepted for publication.

[4] IOE Entrance Exam Result Records since 2012

[5] IOE Semester Exam Result Records since 2012

[6] Lamport L (1978), Time, Clocks and the Ordering of the Events in a Distributed System, Communications of the ACM, 21(7): 558-565 